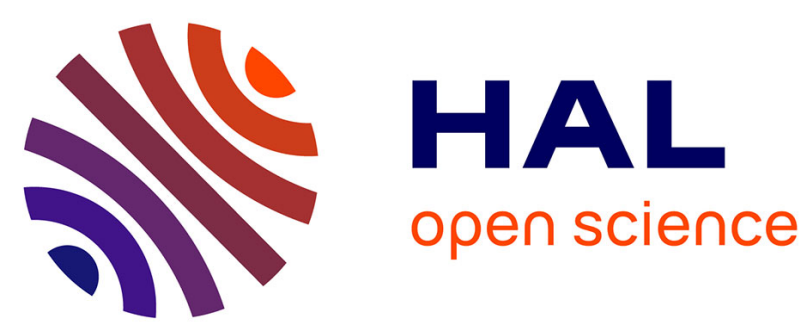

\title{
FAK regulates dynein localisation and cell polarity in migrating mouse fibroblasts
}

Marta Fructuoso, Marlène Legrand, Antoine Mousson, Tania Steffan, Romain Vauchelles, Jan de Mey, Emilie Sick, Philippe Rondé, Denis Dujardin

\section{- To cite this version:}

Marta Fructuoso, Marlène Legrand, Antoine Mousson, Tania Steffan, Romain Vauchelles, et al.. FAK regulates dynein localisation and cell polarity in migrating mouse fibroblasts. Biology of the Cell, 2020, 112 (2), pp.53-72. 10.1111/boc.201900041 . hal-03003641

HAL Id: hal-03003641

https://hal.science/hal-03003641

Submitted on 5 Jan 2021

HAL is a multi-disciplinary open access archive for the deposit and dissemination of scientific research documents, whether they are published or not. The documents may come from teaching and research institutions in France or abroad, or from public or private research centers.
L'archive ouverte pluridisciplinaire HAL, est destinée au dépôt et à la diffusion de documents scientifiques de niveau recherche, publiés ou non, émanant des établissements d'enseignement et de recherche français ou étrangers, des laboratoires publics ou privés. 


\section{FAK Regulates Dynein Localization and Cell Polarity in Migrating Mouse Fibroblasts}

Marta Fructuoso $^{\dagger \ddagger}$, Marlène Legrand ${ }^{\dagger}$, Antoine Mousson $^{\dagger}$, Tania Steffan $^{\dagger}$, Romain $^{\dagger}$ Vauchelles $^{\dagger}$, Jan De Mey ${ }^{\dagger}$, Emilie Sick ${ }^{\dagger}$, Philippe Rondé ${ }^{\dagger}$, and Denis Dujardin ${ }^{\dagger *}$.

${ }^{\dagger}$ Migration, invasion and microenvironnement, Faculté de Pharmacie, UMR7021 CNRS, LBP, Université de Strasbourg, Illkirch, France.

‡ ICM Institut du Cerveau et de la Moelle épinière, CNRS UMR7225, INSERM U1127, UPMC, Hôpital de la Pitié-Salpêtrière, Paris, France.

Running title: dynein and FAK orient the centrosome

*Corresponding author: denis.dujardin@unistra.fr

Phone : $33(0) 68854289$

Fax : $33(0) 68854313$

Key words : cell migration, centrosome polarity, cytoplasmic dynein, FAK 


\section{Abstract:}

\section{Background}

Fibroblasts executing directional migration position their centrosome, and their Golgi apparatus, in front of the nucleus toward the cell leading edge. Centrosome positioning relative to the nucleus has been associated to mechanical forces exerted on the centrosome by the microtubule-dependent molecular motor cytoplasmic dynein 1, and to nuclear movements such as rearward displacement and rotation events. Dynein has been proposed to regulate the position of the centrosome by exerting pulling forces on microtubules from the cell leading edge, where the motor is enriched during migration. However, the mechanism explaining how dynein acts at the front of the cells has not been elucidated.

Results

We present here results showing that the protein Focal Adhesion Kinase (FAK) interacts with dynein and regulates the enrichment of the dynein/dynactin complex at focal adhesions at the cell the leading edge of migrating fibroblasts. This suggests that focal adhesions provide anchoring sites for dynein during the polarization process. In support of this, we present evidence indicating that the interaction between FAK and dynein, which is regulated by the phosphorylation of FAK on its Ser732 residue, is required for proper centrosome positioning. Our results further show that the polarization of the centrosome can occur independently of nuclear movements. Although FAK regulates both nuclear and centrosome motilities, downregulating the interaction between FAK and dynein affects only the nuclear independent polarization of the centrosome.

\section{Conclusion}

Our work highlights the role of FAK as a key player in the regulation of several aspects of cell polarity. We thus propose a model in which the transient localization of dynein with focal adhesions provides a tunable mechanism to bias dynein traction forces on microtubules allowing proper centrosome positioning in front of the nucleus.

\section{Significance}

We unravel here a new role for the cancer therapeutic target FAK in the regulation of cell morphogenesis. 


\section{Introduction}

The ability of cells to adapt their organization in response to their microenvironment requires complex regulatory and mechanotransduction mechanisms that are crucial for proper cell differentiation and function. One illustration of such mechanisms is the acquisition of a polarized organization of the cytoskeleton during cell migration. In many cell types grown on 2-dimensional extracellular matrixes, the centrosome as well as its associated microtubules and membranous organelles, are positioned in front of the nucleus, toward the leading edge of cells (Elric and Etienne-Manneville, 2014; Luxton and Gundersen, 2011). This polarization is at the crossroad of several signaling pathways including non-canonical Wnt, Cdc42/Par6/aPKC, as well as integrin-dependent pathways (Etienne-Manneville and Hall, 2001; Gomes et al., 2005; Palazzo et al., 2001; Schlessinger et al., 2007).

Sharing some similarities with mitotic spindle orientation during cell division, centrosome positioning during interphase is affected by pulling forces on microtubules from the cell cortex as elegantly revealed by the effects of local delivery of a microtubule depolymerizing drug (Burakov et al., 2003). Moreover, centrosome orientation during migration was shown to require the molecular motor cytoplasmic dynein 1 (hereafter referred to as "dynein") and its associated complex dynactin in fibroblasts, astrocytes, and CHO cells (Etienne-Manneville and Hall, 2001; Palazzo et al., 2001; Yvon et al., 2002). In mouse fibroblasts, dynein has been proposed to regulate the position of the centrosome by exerting pulling forces on microtubules from the cell leading edge, where the motor is enriched during migration (Dujardin et al., 2003). The motor was also shown to be functioning at cell-cell contact cortical sites containing Par3 (Schmoranzer et al., 2009), where it was also proposed to tether and/or pull on microtubules, thus achieving a force equilibrium with front edge traction forces leading to centrosome positioning. How dynein is anchored at the leading edge of cells still remains speculative. An interesting possibility is raised by the interaction between the focal adhesion proteins FAK and paxillin with dynein shown in endothelial and NRK cells (Park et al., 2009; Rosse et al., 2012). Moreover, FAK was reported to be required for proper centrosome positioning in mouse fibroblasts (Tomar et al., 2009) as well as other cell types (Maninova et al., 2013; Serrels et al., 2010; Tilghman et al., 2005). However, whether dynein interacts with FAK and paxillin in fibroblasts is still unknown, and the role of these interactions during polarization was never investigated.

In addition, dynein was proposed to be anchored to the nucleus of mice neuronal cells via the SUN1/2 and Syne/Nesprin-1/2 complexes (Zhang et al., 2009). Interestingly, centrosome orientation was linked to the intra cellular behavior of the nucleus. Indeed, in mouse fibroblasts, centrosome polarization was shown to rely on the actin- and Nesprin2G/SUN2dependent rearward movement of the nucleus (Gomes et al., 2005; Luxton et al., 2011). Defects in centrosome positioning were correlated with nuclear rotation (NR) inhibition after disruption of the SUN-Nesprin interaction within the LINC (Linker of Nucleoskeleton and Cytoskeleton) complex in RAT 2 fibroblasts (Maninova et al., 2013). Regulating nuclear positioning, orientation, and shape are thought to help cells migrating in a $3 \mathrm{D}$ environment to cope with narrow constraints imposed by the extracellular matrix (Friedl et al., 2011), and was also proposed to promote centrosome/Golgi polarization (Maninova et al., 2014). However, in 2D cultured fibroblasts, NR depends on dynein and can be observed without affecting the position of the centrosome (Gerashchenko et al., 2009; Levy and Holzbaur, 2008). Thus, to what extent NR could promote centrosome orientation remains unclear. 
In this study, we provide evidences suggesting that focal adhesions are transient anchoring sites for dynein at the leading edge of migrating fibroblasts. We show that dynein interacts with the focal adhesion proteins FAK and paxillin, and that FAK is required for the proper enrichment of dynein and its cofactor dynactin at the leading edge of cells. Our data also show that FAK is required for the dynamic colocalization of dynactin spots with focal adhesions at the cell leading edge. Further functional tests indicate that the regulation of the interaction of FAK with dynein affects the polarization of the centrosome and associated Golgi apparatus. Finally, we clarify the role of different nuclear behavior during polarization, and show that the interaction of FAK with dynein allows centrosome polarization independently of nuclear motility.

\section{Results}

\section{FAK-Dynein interaction regulates Golgi orientation}

To test for the requirement of FAK and its interaction with dynein in the positioning of the centrosome and the Golgi apparatus in front of the nucleus during migration, we compared the ability of mouse fibroblasts, expressing wt and mutated FAK constructs, to polarize. To do so, we used the wound healing assay, in which all cells move homogeneously toward the wound and polarize efficiently within a couple of hours of migration (Palazzo et al., 2001). To get a precise measurement of the efficiency of polarization, we measured the "polarity angle" $\alpha$ (Figure 1A) in migrating fibroblasts fixed and processed for immunofluorescence $4 \mathrm{~h}$ after wounding. Low angles are indicative of well polarized cells, and angles up to $180^{\circ}$ indicate defaults in centrosome/Golgi orientation. $F A K^{--}$Mouse Embryo Fibroblasts (MEF) cells display significantly higher polarization angles $\left(54.1 \pm 4.8^{\circ}\right.$; Figures $1 \mathrm{~A}$ and $\left.1 \mathrm{~B}\right)$ as compared to two MEF controls referred to as wt MEF $\left(35.6 \pm 3^{\circ}\right)$ or NIH 3 T3 fibroblasts $\left(19.2 \pm 0.7^{\circ}\right)$. This polarization defect is specific to FAK as indicated by the rescue of a low polarization angle $\left(26.8 \pm 4.9^{\circ}\right)$ in $\mathrm{FAK}^{-/-}$fibroblasts transfected by a plasmid encoding for FAK tagged with EGFP. Similar results were obtained using $\gamma$-tubulin as centrosome marker to measure the polarity angle (Figure 1A and data not shown).

The interaction of the molecular motor dynein with FAK was already reported in endothelial cells, and its inhibition using the mutated FAK S732A form was shown to trigger mitotic defects (Park et al., 2009). Therefore, to test whether FAK association with dynein is important for Golgi polarization, we constructed a version of FAK fused to EGFP harboring the non-phosphorylatable S732A mutation and expressed it in MEF $\mathrm{FAK}^{-/-}$cells. FAK activation was not affected by the S732A mutation as shown by ratiometric analysis of Y397FAK vs. total FAK in both cell extracts (Figures S1A and S1B), and individual focal adhesions (Figures S1C, S1D, and S1E). Moreover, tyrosine phosphorylation of Y861 and Y925, two major binding sites for protein such as p130CAS, paxillin, or Grb2 (Mousson et al., 2018), were not affected by S732A mutation (Figures S1A and S1B), suggesting that this mutation does not have major impact on FAK structure. FAK S732A-EGFP expressing cells failed to restitute proper Golgi orientation, as indicated by their significantly higher mean angle of polarization (Figures $1 \mathrm{~A}$ and $1 \mathrm{~B} ; 44.3 \pm 3.9^{\circ}$ ), compared to cells expressing FAKEGFP $\left(26.8 \pm 4.9^{\circ}\right)$. To evaluate the proportion of cells able to polarize, we used a polarity angle limit of $45^{\circ}$ as a cut off value (Figure 1C). As previously reported (Tomar et al., 2009), around $80 \%$ of cells are polarized when expressing endogenous FAK or after FAK reintroduction in $\mathrm{FAK}^{-/-}$cells. This value decreases significantly in $\mathrm{FAK}^{-/-}$cells $(54.3 \pm 4.2 \%)$ and FAK S732A-EGFP expressing cells $(64.5 \pm 6.2 \%)$ confirming the requirement of FAK for proper centrosome and Golgi positioning, and suggesting that FAK interaction with dynein is also necessary. 
To confirm that the observed polarization defects were linked to the altered interaction of FAK with dynein, we performed immunoprecipitation assays. Our results show that endogenous FAK and dynein co-immunoprecipitate in MEFs cell extracts using either antidynein intermediate chain (DIC), or anti-FAK antibodies (Figure 2A). We also observed that the co-immunoprecipitation of dynein with paxillin decreases markedly in MEF $\mathrm{FAK}^{-/}$, suggesting that FAK regulates the interaction between dynein and paxillin. To verify whether the S732A mutation affects the interaction between FAK and dynein in MEFs, endogenous DIC was co-immunoprecipitated in cells expressing either FAK S732A-EGFP or FAK-EGFP. As expected, we observed that the S732A mutation induces a significant $50 \%$ decrease of the interaction of FAK with dynein in mouse fibroblasts (Figures $2 \mathrm{~B}$ and $2 \mathrm{C}$ ). Together, these results suggest that the regulation of FAK interaction with dynein is required for proper Golgi positioning.

\section{FAK regulates the enrichment of dynein as well as other cell polarization markers at the leading edge of migrating fibroblasts}

Traction forces from the cell cortex exerted by dynein were proposed to be responsible for centrosome positioning in migrating mouse fibroblasts (Dujardin et al., 2003; Schmoranzer et al., 2009). As dynein was shown to be enriched at the leading edge of fibroblasts (Dujardin et al., 2003), we tested whether FAK affects the localization of dynein during migration. To do so, we performed immunofluorescence experiments to compare the localization of dynein in cells expressing FAK or not (Figure 3A). Measuring the ratio between the mean dynein signal at the leading edge and the mean dynein signal in the whole cell reveals an approximately twofold enrichment of dynein at the leading edge in wt MEFs $(2.2 \pm 0.6, n=30)$, that is lost in FAK $^{-/-}$cells $(1.1 \pm 0.2, n=30$; Figure $3 \mathrm{~B})$. This result, along with the important decrease of the proportion of cells displaying dynein enrichment at the leading edge in $\mathrm{FAK}^{-/-}$cells $(5.5 \pm$ $1.9 \%, n=300)$ compared to wt MEFs $(68.2 \pm 2.4 \%, n=300$; Figure 3C), clearly show that FAK is required for dynein enrichment at the leading edge as visualized by both standard (EPI) or TIRF microscopy. This suggests that FAK regulates the localization of dynein during cell migration and polarization. FAK removal did not affect the amount of the motor in cell extracts (Figure S1F) indicating that the absence of dynein at the leading edge of $\mathrm{FAK}^{-/-}$cell is not due to a down regulation of dynein synthesis.

Dynein is also required for centrosome polarization in astrocytes where its interaction with microtubules at the protrusion of migrating cells depends on its association with GKAP (Guanylate Kinase-Associated Protein) protein (Elric and Etienne-Manneville, 2014; Manneville et al., 2010). In this cell type, the APC (Adenomatous Polyposis Coli) tumor suppressor also affects centrosome orientation and could act as another microtubule anchoring system at the cell leading edge (Etienne-Manneville et al., 2005). However, APC was showed to affect only microtubule stabilization in mouse fibroblasts (Wen et al., 2004). To determine whether FAK also affects the localization of these proteins, we performed immunofluorescence experiments to detect APC and GKAP proteins. Our results show that APC and GKAP are enriched at the leading edge of migrating fibroblasts as compared to whole cells (Figure 3A). The analysis of this phenotype revealed that $80.9 \pm 4.2 \%$ and $85.7 \pm$ $13.4 \%$ of wt MEF are enriched in APC and GKAP respectively ( $\mathrm{n} \geq 300$ randomly chosen cells for each condition). These values dropped to $10 \pm 2.6 \%$ and $13.4 \%$ in $\mathrm{FAK}^{-/-}$cells (Figure 3C). These enrichments are mostly visible using TIRF microscopy indicating that they are mainly located at the base of cells. Moreover, precise measurement of the ratio between the mean APC or GKAP signals at the leading edge and the mean APC or GKAP signals in 
whole wt MEFs reveals an approximately twofold enrichment of APC (2.6 \pm 0.8$)$ and GKAP $(2.2 \pm 0.6)$ at the leading edge (Figure 3B, n=30). As for dynein, these enrichments are strongly inhibited in $\mathrm{FAK}^{-/-}$MEFs (APC : $1.1 \pm 0.2$; GKAP : $1.2 \pm 0.2$ ), with no effect on APC and GKAP synthesis (Figure S1F). The modification of the distribution of APC in FAK ${ }^{-}$ I- fibroblast is consistent with the decreased stabilization of microtubules monitored in the same cell type (Palazzo et al., 2004). However, performing co-immunostaining experiments for GKAP and dynein intermediate chain, no obvious colocalization between the two proteins were detected. Indeed, they appeared to be enriched at different sub-locations of the cells leading edges (Figure 3D). Thus, it remains uncertain whether GKAP and dynein are part of a complex in mouse fibroblasts, as proposed in astrocytes (Manneville et al., 2010; Melkonian et al., 2007). To analyze dynein enrichment at the leading edge of living cells, we coexpressed EGFP and mCherry tagged versions of DIC and the focal adhesion marker vinculin in mouse fibroblasts. Cells co-expressing DIC and vinculin and located at the wound edge were monitored by TIRF microscopy within 3 hours after wounding. Time laps sequences reveal an enrichment of dynein spots at the front of cells in $90 \%$ of NIH $3 \mathrm{~T} 3(n=15)$ or wt MEFs ( $n=14)$ cells (Figure 3E top rows, white and green arrows), consistent with the enrichment of the motor observed by immunofluorescence. The spots appeared very dynamic and persisted only several seconds (Movie S2). Consistent with our previous results, such enrichment were not observed in $\mathrm{FAK}^{-/-}$cells $(\mathrm{n}=20$, Figure $3 \mathrm{E}$ and Movie S3). Moreover, immunofluorescence experiments indicate that $85 \%$ of EGFP spots associate with microtubules in FAK expressing fibroblasts (Figure 3E middle left row, arrows, $n=250$ spots from 13 cells), which is consistent with previously reported colocalization of dynein with microtubules (Dujardin et al., 2003). Also, $97 \%$ of the spots appeared to be present at the extremity of actin stress fibers possibly ending in focal adhesions (Figure 3E middle right row, see arrows, $n=275$ spots from 9 cells). However, no clear colocalization between dynein and focal adhesion was previously detected in fixed fibroblasts (Dujardin et al., 2003). On the other hand, overexpressed p62 subunit of dynactin or dynein intermediate chain 2 were detected at focal adhesions in COS7 and NRK cells (Garces et al., 1999; Rosse et al., 2012), and our experiments in living cells show DIC spots localized on, or in close proximity to focal adhesions (Figure 3E, top row and associated magnified inserts and line intensity profile measurement). This suggests that the association of dynein with FAK may be transient. To test this hypothesis, we performed TIRF video microscopy on cells co-expressing mCherry tagged vinculin, and low levels of EGFP tagged dynamitin, a subunit of the dynein cofactor dynactin that colocalizes with dynein at the leading edge (Dujardin et al., 2003) and is required for cell polarization (Palazzo et al., 2001). The distribution of dynamitin resembles that of dynein, with more than $94 \%$ of the spots associated with microtubules (Figure 3E bottom left row; $n=375$ spots from 30 FAK expressing cells), and $90 \%$ of the spots associated with the extremity of stress fibers (Figure 3E bottom right row; $\mathrm{n}=111$ spots from 17 cells). As for dynein, a clear enrichment of dynactin spots at the front of $95 \%$ of living cells was observed ( $\mathrm{n}>50$ cells, Figure 4A upper panels). These spots appeared very dynamic and persisted only several seconds (Movie S4). The distribution of the dynactin spots changed markedly in $\mathrm{FAK}^{-/-} \mathrm{MEFs}$, where no enrichment at the front of these cells was detected (Figure 4A lower panels, Movie S5). Quantification of the percentage of cell base surface displaying dynamitin staining at the front or the rear of cells, reveals a strong and statistically significant enrichment of dynactin at the front of living migrating cells that is lost in $\mathrm{FAK}^{-/-}$ MEFs (Figure 4B). These results are consistent with a role for FAK in regulating a polarized distribution of dynein and its cofactors at the front of migrating cells. To determine whether this enrichment is localized at focal adhesions, we next analyzed the percentage of cell base surface where the dynactin signal colocalizes with vinculin at the front or at the rear of cells. Figure $4 \mathrm{C}$ shows a strong and significant enrichment of dynactin colocalization with focal 
adhesions at the front of MEFs that is lost in $\mathrm{FAK}^{-/-}$MEFs. This suggests that FAK is required to locally increase the frequency of transient associations between dynactin and focal adhesions. To further investigate this aspect, we measured the persistence or "dwell time" of colocalizing spots. Kymographs clearly show longer stretches of colocalizing signal at the front of wt MEFs (Figure 4D white lines and arrow) that are less frequent at the rear (Figure 4E). Again, this front/rear asymmetric behavior is lost in $\mathrm{FAK}^{-/-} \mathrm{MEFs}$. On the other hand, expressing FAK-mCherry in $\mathrm{FAK}^{-/}$MEFs led to a rescue of the Front/Rear asymmetry (Figures 4B, 4C, 4D, 4E and Movie S6). Together, our results show a new role for FAK in regulating the front/rear asymmetry of colocalization and dwell time of dynactin clusters at focal adhesions, and are consistent with FAK regulation of dynein functions at frontal focal adhesions.

\section{FAK and dynein regulate Golgi positioning independently of nuclear behavior}

The position of the centrosome and associated Golgi apparatus in front of the nucleus can theoretically result from the intracellular motion of the centrosome and/or the displacement of the nucleus itself. Indeed, in non-migrating mouse fibroblasts induced to polarize by lysophosphatidic acid, an actin- and nesprin2G/SUN2-dependent rearward nucleus movement responsible for the orientation of the centrosome was observed (Gomes et al., 2005; Luxton et al., 2010). These observations highlighted an initial nuclear dependent polarization event that occurs before the onset of migration. Nuclear rotation events were also proposed to promote Golgi polarization toward the front of cells (Maninova et al., 2013). We then explored whether Golgi motion is linked to these different nuclear behaviors in migrating fibroblasts, and whether FAK is involved in their regulation. For this purpose, we tracked both the Golgi apparatus and the nucleus in living cells during polarization. Confluent fibroblasts expressing, or not, FAK constructs along with fluorescent Golgi markers were wounded, and nonpolarized cells at the migratory front were monitored by time lapse microcopy within 30 minutes after wounding.

We observed that in most FAK expressing cells, the nucleus is already positioned at or close to the rear of cells at the beginning of the monitoring, and that complete Golgi polarization takes place subsequently, as illustrated in Figure 5A (upper panel, Movie S7). This observation confirms that the nuclear rearward motion is an early process, but it also indicates that it is not sufficient to achieve proper Golgi polarization in migrating fibroblasts. To evaluate the evolution of the position of the nucleus, we recorded the coordinates of the cell rear and cell front as well as the coordinates of nucleus centroid (Figure 5A). This allowed calculating the position of the nucleus as a percentage of total cell length (0 to $100 \%$ representing the back and the front respectively) before and after polarization. This quantification shows a limited but reproducible mean nuclear rearward displacement of approximatively $10 \%$ of cell length in NIH3T3, wt MEFs, or FAK-EGFP expressing cells (Figure 5B). In $\mathrm{FAK}^{-/-}$cells, this movement is inhibited and nuclei are allowed to move forward during cell protrusion toward the wound $(2.17 \pm 13.4 \%$; Figure $5 \mathrm{~A}$ and associated Movie S8, Figure 5B). This indicates that FAK is required for proper nuclear positioning. Interestingly, this positioning is independent of FAK interaction with dynein. Indeed, the rearward positioning of the nucleus is not altered in FAK S732A-EGFP expressing cells (Figures 5A and 5B, and associated movie S9). Monitoring the position of the Golgi apparatus in the same cells (Figure 5A) reveals a frontward movement in opposite direction of the nuclei in FAK expressing cells (approximately $10 \%$ of cell length, Figure 5C). Consistent with our results on fixed cells, the movement of the Golgi toward the front of cells is inhibited in FAK /- cells with a rather rearward displacement $(-8.1 \pm 17.4 \%)$. However, FAK S732A-EGFP expressing cells displayed a very limited frontward displacement $(6.7 \pm 19.1 \%)$ not 
significantly different from FAK expressing cells, suggesting the Golgi is able to move frontward when FAK interaction with dynein is downregulated. Nevertheless, analyzing the percentages of polarizing cells using the same dataset indicates a defect in Golgi polarization in FAK S732A-EGFP expressing cells (Figure 5D), as observed in fixed cells (Figure 1). These results suggest that other types of displacement, such as rotational events around the nucleus, may be necessary to fully polarize the Golgi.

We next evaluated the occurrence of Nuclear Rotations (NRs) in our cell models. Nucleoli observed by phase contrast microscopy were used as intranuclear markers to monitor NRs that were frequently observed during the migration process (Figure 6A, Movie S10). Quantifying the percentage of cells displaying NR events during the time frame of the experiment revealed that 44 to $62 \%$ of FAK expressing cells displayed such behaviors. This frequency is significantly decreased in $\mathrm{FAK}^{-/}$cells (Movie S8), but not in FAK S732A-EGFP expressing cells compared to wt MEFs and FAK-EGFP control cells, indicating that FAK, but not its interaction with dynein, is required for NR (Figure 6B, see also Movies S11 and S12 for respectively FAK-EGFP and FAK S732A-EGFP expressing cells). To evaluate the contribution of NR events to the positioning of the Golgi apparatus, we monitored changes in Golgi orientation (Figure 7A red arrows) relative to NR events (yellow arrows) in the same dataset. Time-course analysis of both nucleus and Golgi angle revealed that persistent Golgi rotation events (superior to $10^{\circ}$ and lasting more than 3 frames) were either accompanied with simultaneous rotation of the nucleus in the same direction (Figure 7A) or occurred completely independently of NR (Figure 8A). Quantification of these events has shown that Golgi polarization occurs simultaneously to nuclear rotation in 40 to $50 \%$ of the cells expressing FAK or FAK-EGFP (Figure 7A, see also corresponding Movies S10 and S11). Consistent with previous results showing that FAK is required for NR, the removal of FAK significantly decreases the frequency of NR linked polarization (Figure 7, Movie S8). Interestingly, our results show that downregulating the interaction of dynein with FAK via the expression of FAK S732A-EGFP does not affect the polarization associated with NR (Figures 7A and 7B, Movie S12). Most interestingly, Golgi polarization was observed to occur independently of NR in approximately $50 \%$ of FAK expressing cells (Figures 8A and 8B, compare the amplitudes of red and yellow arrows as well as the independent evolution of Golgi and nucleus angles; see also corresponding Movies S7 and S13 for respectively NIH 3T3 and FAK-EGFP expressing MEFs). FAK is required for NR-independent orientation of the Golgi as indicated by the significant decrease of its frequency in $\mathrm{FAK}^{-/}$cells (Figure 8B, see Figure 7A, Movie S8, for typical behavior illustration). Moreover, FAK S732A-EGFP expressing cells also displayed a strong inhibition of NR independent frequencies (Figure 8B), and Golgi orientations were almost seen uniquely associated with NR (Figure 7A, Movie S12). Our observations thus indicate that the regulation of FAK association with dynein is required for the positioning of the Golgi apparatus independently of nuclear motility. 


\section{Discussion}

The positioning of the centrosome, and thus most of the microtubule network, in front of the nucleus, was proposed to enhance cell migration by facilitating polarized membrane trafficking with the leading edge (Etienne-Manneville, 2013; Luxton and Gundersen, 2011). This asymmetric distribution of microtubules also polarizes Rho and Rac GTPase signaling (Krendel et al., 2002; Waterman-Storer et al., 1999), which regulates cell protrusion and cell adhesion. Microtubules interaction with focal adhesion was already shown to regulate microtubules dynamics (Efimov et al., 2008; Small and Kaverina, 2003), as well as focal adhesion disassembly (Ezratty et al., 2005; Kaverina et al., 1999; Stehbens and Wittmann, 2012). Here we show that FAK regulates the orientation of the centrosome and associated Golgi apparatus in mouse fibroblasts as well as the polarized enrichment of dynein and dynactin at the leading edge. Moreover, IP experiments show that FAK and paxillin interact with dynein in mouse fibroblasts, consistent with the interaction between dynein intermediate chain 2, paxillin, and FAK reported in mouse endothelial and NRK cells (Park et al., 2009; Rosse et al., 2012). These results indicate that focal adhesions could provide anchoring sites for dynein to exert traction forces on microtubules resulting in forward centrosome positioning relative to the nucleus. In agreement with this hypothesis, downregulating the interaction between dynein and FAK, by preventing its phosphorylation on residue 732 (Park et al., 2009), impaired Golgi polarization. We observed that this mutation did not abolish completely the interaction between FAK and dynein. This suggests that this interaction may not rely only the S732 residue, and could also be indirect involving other proteins. Consistent with this hypothesis, we observed that dynein also coimunoprecipitates with paxillin. The restriction of FAK S732 phosphorylation to a subset of focal adhesions at the leading edge could be a possible way to upregulate dynein based pulling forces on the microtubules at the front of cells. Indeed, inhibiting the formation and function of the FAK/RACK1/PDE4D complex was shown to prevent nascent adhesions stabilization as well as Golgi polarization (Serrels et al., 2011; Serrels et al., 2010). Moreover, we were able to highlight a dynamic and FAK dependent concentration of EGFP tagged dynein and dynactin spots at focal adhesions located at the front of migrating cells. These structures appeared to codistribute with microtubules, consistent with previously reported colocalization using immunofluorescence on endogenous proteins in fibroblasts (Dujardin et al., 2003) and astrocytes (Manneville et al., 2010). However, in astrocytes, dynein was shown to be responsible for the bending of microtubules toward the cell base at a location behind the focal adhesion-rich adhesive region (Manneville et al., 2010). This observation differs from the distribution of dynein we observed in fibroblasts and may represent mechanistic differences between astrocytes and fibroblasts. In our cell model, the increased attractiveness and dwell time of dynactin at cell front focal adhesions could reflect an enrichment of dynein traction activity on microtubules capable of moving the centrosome ahead. Thus, a picture emerges where FAK appears to be an essential mediator of the polarization process via both formation and stabilization of nascent adhesions (Deramaudt et al., 2011; Serrels et al., 2011), which then could serve as anchor points to promote dynein-dependent centrosome orientation. Both the FAK dependent behavior of dynein at focal adhesion, and the coimmunoprecipitation between dynein and FAK and paxillin, points to focal adhesions as obvious candidates to anchor dynein. However we cannot rule out that other cortical structures could also be involved in the polarization of the centrosome. Indeed, the positioning of the centrosome inside the cell is expected to be influenced by several dynein pulling forces acting in opposite directions. Nuclear envelope associated dynein would act to keep the centrosome close to the nucleus (Robinson et al., 1999; Zhang et al., 2009). Meanwhile, Dynein forces from cell to cell contact sites located at the rear of cells during a wound healing assay would also bias centrosome positioning to the 
rear (Schmoranzer et al., 2009). Thus, the regulation of the association of dynein with cell front focal adhesions via FAK could provide the fibroblasts with a tunable mechanism to adjust centrosome and Golgi central positioning during migration.

Nuclear rotation events, leading to the alignment of the longer nuclear axis with the direction of migration, have been proposed to promote also centrosome orientation (Luxton and Gundersen, 2011; Maninova et al., 2014). Indeed, in mouse fibroblasts subjected to mechanical shear stress, both nucleus rotation and centrosome orientation are controlled via Cdc42 (Lee et al., 2005), and both depend on the molecular motor dynein in wound healing assays (Etienne-Manneville and Hall, 2001; Levy and Holzbaur, 2008; Palazzo et al., 2001). Moreover, interfering with the connection between the cell cytoskeleton and the nuclear lamina resulted in both nuclear as well as centrosome disorientation (Houben et al., 2009; Lee et al., 2007; Luxton et al., 2010; Maninova et al., 2013; Osorio and Gomes, 2014). These results, together with our observations indicating that nuclear and Golgi orientation take place simultaneously, are strongly in favor of non-coincidental orientation events. Centrosome orientation and nuclear rotation were both shown to depend on FAK kinase activity and its substrate p190RhoGAP (Maninova et al., 2013; Tomar et al., 2009) supporting the idea of a common mechanism linking both phenomena. Consistent with that, we report here that $\mathrm{FAK}^{-/-}$ cells display lower frequencies of NRs than FAK expressing cells, a phenotype that can be rescued by transfecting cells with FAK-EGFP. Interestingly, normal NR frequencies are also rescued after transfecting $\mathrm{FAK}^{-/-}$fibroblasts with the FAK S732A construct, suggesting that FAK interaction with dynein is not essential for NR. This correlates well with the fact that NR was reported to be independent of dynactin localization at the leading edge (Levy and Holzbaur, 2008), and suggest that other anchoring points, such as nuclear envelope or cell-cell contact cortical sites (Burakov and Nadezhdina, 2013; Schmoranzer et al., 2009), could allow dynein to power NR. The requirement of an intact connection between the nuclear lamina and the cell cytoskeleton for proper centrosome positioning (Maninova et al., 2014) and the ability of cells to operate NR rotation without affecting centrosome positioning (Levy and Holzbaur, 2008) could at first sight appear to be contradictory. However, we show here that centrosome orientation can happen both associated and independently of NR in a same cell. Regulating the interaction of FAK with dynein could then modulate the behavior of the centrosome, being capable of following the nucleus as a passenger during NR, or having its own independent motion ensuring to acquire and maintain its polarization during cell migration. FAK S732 phosphorylation, which was shown to depend on CDK5 and VEGF/ROCK signaling in cultured neocortical neurons and endothelial cells (Le Boeuf et al., 2006; Xie and Tsai, 2004), thus appears to be an important target to regulate nuclear independent centrosome motility.

During migration, the centrosome can be oriented at the front or at the back of the nucleus depending on the cell type (Luxton and Gundersen, 2011; Yvon et al., 2002) or, surprisingly, the nature and geometry of their extracellular matrix (Doyle et al., 2009; Pouthas et al., 2008; Schutze et al., 1991). Our results predict that modifying the distribution of focal adhesions in cells is susceptible to affect centrosome positioning by affecting dynein anchoring points. Indeed, fibroblasts have been shown to switch between front or rear centrosome polarization depending on the $2 \mathrm{D}$ or $3 \mathrm{D}$ structure of their extracellular matrix, which affected FAK and paxillin intracellular localization (Cukierman et al., 2001). Fibroblasts are also capable of sensing the direction of the mechanical stress provided by a shear flow to orient their centrosome (Lee et al., 2005), which is consistent with the implication of mechanosensors in the regulation of cell polarity. Accordingly, focal adhesions and especially FAK are part of mechanosensing mechanisms allowing cells to sense the physical characteristics of the microenvironment to regulate cell polarity (Plotnikov et al., 2012; Prager-Khoutorsky et al., 
2011; Wang et al., 2001), and a role for FAK as a mechanosensor affected during the progression of cancer was already proposed (Tilghman and Parsons, 2008). The characterization of how dynein functions are integrated in mechanotransduction signaling pathways during centrosome positioning remain important and challenging points to investigate. 


\section{Materials and Methods}

\section{Plasmids:}

The plasmids encoding for the following fusion proteins were kindly provided by the following researchers: mCherry-Galactosyltransferase (mCherry-GT) (Miller et al., 2009) was provided by I. Kaverina (Vanderbilt University Medical Center, Nashville, USA), 2Nacetylglucosaminyltransferase I (NAGFP)(Shima et al., 1997) was a gift from D. Shima and G. Warren (Imperial Cancer Research Fund, London, UK), dynamitin-EGFP (Dohner et al., 2002) is from the laboratory of R. Vallee (Columbia University, NY, USA), and mCherryvinculin (Wolfenson et al., 2009) was provided by I. Lavelin (Weizmann Institute of Science, Rehovot, Israel). Mouse EGFP-tagged dynein intermediate chain IC2C (DIC) was obtained from Addgene (King et al., 2003). Human wild-type FAK fused to C-terminus of EGFP or mCherry are described in (Deramaudt et al., 2014). FAK-EGFP plasmid was used as template to generate the S732A and S732D substitution constructs with the QuikChange II XL sitedirected mutagenesis kit (Agilent Technologies). The following sequences were used to design the primers used for the PCR reactions: S732A: 5'GCGAAGGATTTTATCCCGCTCCACAGCACATGGTAC-3'; S732D: 5'GCGAAGGATTTTATCCCGACCCACAGCACATGGTAC-3'. All constructs were amplified and purified using Qiagen Hispeed Maxiprep kits and verified by sequencing.

\section{Cell culture and transfection:}

NIH3T3 fibroblasts (Gomes et al., 2005), primary $\mathrm{FAK}^{-/}$mouse embryonic fibroblasts (MEFs) (Illc et al., 1995), and wild-type (wt) MEFs (gift from Dr. P. Boucher, UMR7213, Illkirch, France), were maintained at $37^{\circ} \mathrm{C} 5 \% \mathrm{CO}_{2}$ in DMEM supplemented with $10 \%$ Fetal Bovine Serum, $100 \mathrm{U} / \mathrm{ml}$ penicillin and $100 \mu \mathrm{g} / \mathrm{ml}$ streptomycin (Gibco) and tested for contamination. Cells were seeded to reach confluency in $48 \mathrm{~h}$, either on cell culture petri dishes to prepare cell extracts, or on $18 \mathrm{~mm}$ coverslips placed in 6 well dishes for immunofluorescence labeling. Transfections were performed on the day of seeding in 6 well dishes using Lipofectamine 2000 (Invitrogen) according to the manufacturer's directions. For cell polarization assays, confluent cells plated on coverslips were wounded with a pipet tip, and either replaced in the incubator to allow for migration for 4 hours before to be processed for immunofluorescence, or directly mounted in a Ludin Chamber for TIRF and video microcopy analysis (Life Imaging Services, Basel Switzerland).

\section{Immunocytochemistry and immunological reagents}

For immunofluorescence, coverslips were briefly rinsed in $37^{\circ} \mathrm{C}$ PHEM buffer $(120 \mathrm{mM}$ PIPES, $50 \mathrm{mM}$ HEPES, $20 \mathrm{mM}$ EGTA and $4 \mathrm{mM}$ magnesium acetate, $\mathrm{pH}$ 6.9) and fixed with $3 \%$ PFA in PHEM for 20 min followed by permeabilization either during 20 min with $0.5 \%$ TritonX100 in PBS (Figures 1A, 3A GKAP, 3E, and S1C), or during $4 \mathrm{~min}$ in $-20^{\circ} \mathrm{C}$ methanol (Figures 3A DIC and APC). Cells imaged in Figure 3F were fixed in PHEM buffer containing $0.05 \%$ glutaraldehyde, 3\% PFA, and 0.05\% TritonX100 during $12 \mathrm{~min}$, and subsequently incubated 3 times $10 \mathrm{~min}$ in $1 \mathrm{mg} / \mathrm{ml} \mathrm{NaBH} 4$ in PBS to reduce the autofluorecence. Permeabilization was then carried out during 20 min with $0.5 \%$ TritonX100 in PBS. For all experiments, coverslips were then incubated for $30 \mathrm{~min}$ with $0.05 \%$ BSA in PBS and incubated for $1 \mathrm{~h}$ in primary antibody at $37^{\circ} \mathrm{C}$, washed, and incubated for $1 \mathrm{~h}$ in secondary antibodies at $37^{\circ} \mathrm{C}$. After several washes in PBS and a final incubation with DAPI $(0.1 \mu \mathrm{g} / \mathrm{ml}$, Sigma-Aldrich), coverslips aimed at regular fluorescence analysis were mounted using Prolong Gold Antifade Reagent (Life Technologies). Coverslips aimed at TIRF 
microscopy analysis were kept in PBS. Antibodies(and dilution used for immunofluorescence), included mouse Anti-Golgi GM130 (1/200) from BD Transduction Laboratories, mouse anti Dynein Intermediate Chain MAB1618 (1/100) from Millipore, rabbit anti $\gamma$-tubulin (1/300) and Mouse anti $\alpha$-tubulin (DM1A, 1/200) from Sigma, mouse anti APC (Ab-5, 1/50) from Oncogene Research Products, rabbit anti GKAP (SAPAP1, 1/50) from Santa Cruz, and rabbit anti FAK-P397 (1/100) from Invitrogen. Actin was stained using Alexa Fluor 568 phalloidin (1/200; Invitrogen). The antibodies used for western blotting were diluted either ten times further compared to immunofluorescence, or to $1 / 1000$ for the mouse anti FAK from BD Transduction Laboratories, rabbit anti FAK-P861 from Invitrogen, rabbit anti FAK-P925 from Cell Signaling Technologies, and mouse anti Paxillin from BD Transduction Laboratories. Secondary antibodies were goat anti-rabbit or anti-mouse IgG Alexa Fluor 488, 568 and 680 from Life Technologies (1/350), and goat anti-mouse or rabbit IgG HRP conjugate from Bio-Rad (1/10000).

\section{Immunoprecipitation and western blotting}

For standard protein analysis by western blots to check for DIC or FAK constructs expression, cells grown in 6 well dishes and transfected with the proper constructs were washed twice with ice cold PBS and lysed with modified RIPA buffer $(150 \mathrm{mM} \mathrm{NaCl}, 50 \mathrm{mM}$ Tris, $\mathrm{pH} 8.0$, $1 \%$ Triton X100) with Complete Protease Inhibitor Cocktail (Roche). Cells were recovered with a cell scrapper, incubated $20 \mathrm{~min}$ on ice, and spun at $13000 \mathrm{~g}$ for $10 \mathrm{~min}$ in a microfuge (Eppendorf). The supernatants were collected and placed at $95^{\circ} \mathrm{C}$ in $2 \times$ SDS-PAGE sample buffer for $5 \mathrm{~min}$. Samples were loaded into 8\% SDS-PAGE gels and transferred onto Immobilon-P membranes (Millipore). Immunoblotting was performed with various antibodies against specific proteins and revealed with Clarity Western ECL substrate (Bio-Rad) and LAS 4000 imager (GE Healthcare).

For co-immunoprecipitation experiments, the polarization of fibroblasts grown to confluency in petri dishes was induced by applying several wounds to the monolayer, which extent was homogeneously reproduced from one dish to another by using a multichannel pipette, and allowed to migrate during $3 \mathrm{~h}$. Cells were then washed twice with ice cold PBS and lysed with modified RIPA buffer (137 mM NaCl, $20 \mathrm{mM}$ Tris, $\mathrm{pH}$ 8.0, 10\% glycerol, 1\% IGEPAL CA630, 3mM Na3Vo4) with Complete protease inhibitor cocktail (Roche). Cells were then incubated on ice for $30 \mathrm{~min}$, and cell extracts were recovered with a cell scrapper and spun at $13000 \mathrm{~g}$ for $10 \mathrm{~min}$ in a microfuge (Eppendorf). The protein concentration in the extracts was determined using the DC protein assay (Bio-Rad), and adjusted. $1 \mathrm{mg}$ of total protein of precleared extracts were added with $4 \mu \mathrm{g}$ of anti FAK antibodies or $2 \mu \mathrm{g}$ of dynein or paxillin antibodies and incubated $2 \mathrm{~h}$ at $4^{\circ} \mathrm{C}$ with gentle rotation. $75 \mu \mathrm{l}$ of RIPA-washed beads (Fast Flow Protein $\mathrm{G}$ sepharose from GE Healthcare) was then added followed by a $3 \mathrm{~h}$ incubation at $4^{\circ} \mathrm{C}$ with gentle rotation. Beads were then washed 4 times with RIPA, and placed at $95^{\circ} \mathrm{C}$ in $2 \times$ SDS-PAGE sample buffer for $5 \mathrm{~min}$. $30 \mu \mathrm{l}$ samples were loaded into $8 \%$ SDS-PAGE gels and processed for immunoblotting.

\section{Microscopy and live cell imaging}

Immunofluorescence or live cell samples were analyzed using a Leica DMIRE2 microscope equipped with a $37{ }^{\circ} \mathrm{C} 5 \% \mathrm{CO}_{2}$ control system (Life Imaging Services). Live cell nuclei were stained $30 \mathrm{~min}$ with Hoesht-33342 (100 ng/ml; Sigma) before wounding the monolayers. The coordinates of several fields containing transfected cells localized with the EGFP/mCherry signals were memorized using a Märzhäuser (Wetzlar, Germany) automated stage piloted by the Leica FW4000 software. Images were then acquired with a $100 \times$ HCX PL APO (1.4 NA) 
objective (immunofluorescences), or a 40 x HCX PL APO (1.25 NA) objective (time lapse experiments) every 5 min (MEFs), or 10 min (NIH3T3), during $5 \mathrm{~h}$ using a Leica DC350FX CCD camera piloted by the FW4000 software. The A4 (320-380 nm excitation (Ex), $400 \mathrm{~nm}$ beam splitter (Bs), 450-490 nm emission (Em), L5 (460-500 nm Ex, $505 \mathrm{~nm}$ Bs, 512-542 Em), Y3 (510-560 nm Ex, $565 \mathrm{~nm} \mathrm{Bs,} \mathrm{572.5-647.5} \mathrm{Em),} \mathrm{and} \mathrm{Y5} \mathrm{(590-650} \mathrm{nm} \mathrm{Ex,} 660 \mathrm{~nm}$ Bs, 662.5-737.5 Em) Leica filter sets were used for imaging. Time-lapse movies were then analyzed using ImageJ software (National Institutes of Health, USA).

TIRF or dual wide-field-TIRF experiments cells were imaged using an iMIC microscope piloted by the Live Aquisition software L.A. 2.4 (Till Photonics), and equipped with a Cobolt Dual Calypso Laser 491/532 nm, a monochromator Polychrome V, an Olympus 60x TIRFM (1.45 NA) objective, and an EMCCD camera (Andor Technology). EGFP was imaged via a selective 455-495nm Ex, $501 \mathrm{~nm}$ Bs, and 500-551 nm Em filter set. mCherry was imaged via a selective 525-564nm Ex, $573 \mathrm{~nm}$ Bs, and 578-638 nm Em filter set. DAPI was imaged via a four bands Bs equipped with a $427-464 \mathrm{~nm}$ Em filter. Live cells were maintained at $37^{\circ} \mathrm{C}$ in a $5 \% \mathrm{CO}_{2}$ humidified atmosphere using an environmental control system (Life Imaging Services).

\section{Cell polarity analysis and statistics}

The polarity angle $\alpha$ is formed between the polarity axis of the cells, defined by the line passing through the centroid of the nucleus and the centroid of either the Golgi or the centrosome (Figure 1A red line), and the migration axis of the cells (Figure 1A green arrow), defined as perpendicular to the wound axis. $\alpha$ was measured from pictures taken randomly along the wound. We wrote an ImageJ macro allowing for the semi-automatized segmentation of multichannel immunofluorescent pictures to determine the centroids of the Golgi apparatus and of the nuclei. Briefly, the DAPI channel pictures are treated by a 1 pixel radius Gaussian filter and thresholded using the algorithm of ( $\mathrm{Li}$ and Tam, 1998). A watershed mask is then applied on the binary images to help separating the nuclei of neighboring cells. A similar procedure is used for the Golgi image with a 3 pixels radius Gaussian filter and an Otsu thresholding algorithm (Otsu, 1979). The contour of each segmented object is drawn on the picture to allow for the visual verification of the quality of the segmentation process. Proper Golgi/Nuclei pairs are then selected manually. The migration axis is defined as perpendicular to the manually drawn wound axis, which can simply follow the main wound applied to the tissue, or can be defined individually for each cell by tracing a line joining the two last points of contact with its neighboring cells. Same results were obtained using both methods of evaluation (not shown). The polarity angle is then calculated via simple trigonometry using the vector drawn from the centroid of the nuclei and the centroid of the Golgi, and the vector of the migration axis. The vectors are then overlaid on the pictures for visual validation of the measurement.

To analyze the colocalization between dynamitin-EGFP and mCherry-vinculin in live cells, each TIRF image from time-lapse sequences was segmented using another ImageJ based macro. Briefly a Hi-Pass filter was applied to both channels to remove inhomogeneous background coming from cytosolic signal and images were binarized using the ImageJ tool detection of maximas in order to separate connected elements. Threshold values were adjusted to optimize object segmentation and regions of interest (front and rear of cell separated by a line located at $1 / 3^{\text {rd }}$ of total cell length) were hand drawn. The surface of segmented objects and their overlap in-between channels are then measured for each frame and exported in tables, segmented pictures and kymographs. 
Statistical comparisons were based on 3 to 7 independent experiments using either Student ttests for comparing two conditions (Figs. 2, 3, 4, and S1) or one-way ANOVA followed by Turkey's multiple comparison test. Differences were judged significant when $p<0.05$, and significance degrees are indicated on the figures by stars $\left({ }^{*} \mathrm{p}<0.05, * * \mathrm{p}<0.01, * * * \mathrm{p}<0.001\right)$. Error bars in the figures represent standard deviations.

\section{Abbreviations}

Adenomatous Polyposis Coli (APC), Chinese Hamster Ovary (CHO), Cytoplasmic Dynein Intermediate Chain (DIC), Focal Adhesion Kinase (FAK), Guanylate Kinase-Associated Protein (GKAP), Linker of Nucleoskeleton and Cytoskeleton (LINC), Mouse Embryo Fibroblasts (MEF), Nuclear Envelope Spectrin Repeat Proteins (Nesprin), Nuclear Rotation (NR), Phosphodiesterase 4D (PDE4D), Receptor for Activated C Kinase 1 (RACK1), Sad1/UNC-84 (SUN), Total Internal Reflection Fluorescence (TIRF). 


\section{Acknowledgements}

We would like to thank E. Gomes for the gift of NIH3T3 cells, P. Boucher for providing MEF cells, and D. Ilic for the $\mathrm{FAK}^{-/}$cell line. We would also like to thank I. Kaverina for providing the plasmid encoding mCherry-Galactosyltransferase (mCherry-GT), I. Lavelin for the mCherry-vinculin plasmid, D. Shima and G. Warren for the $2 \mathrm{~N}$ acetylglucosaminyltransferase I plasmid, and R.B. Vallee for the dynamitin-EGFP plasmid. We thank the QUEST platform imaging facility.

\section{Funding}

This work was supported in part by grants from the UMR7213 inter-team projects to D.D., the Ligue Contre le Cancer to P.R., and from the Institut National du Cancer to J.D.M.[ INCA 2014-178)].

\section{Competing interests}

The authors declare no competing or financial interests.

\section{Author contribution}

Marta Fructuoso, Marlène Legrand, Antoine Mousson, Tania Steffan, and Denis Dujardin carried out the experimental work. All authors contributed to data analysis and interpretation. Denis Dujardin, Philippe Rondé, Jan De Mey, Marta Fructuoso, and Romain Vauchelles, contributed to the writing of the article. 


\section{References}

Burakov, A., Nadezhdina, E., Slepchenko, B., and Rodionov, V. (2003). Centrosome positioning in interphase cells. J Cell Biol 162, 963-969

Burakov, A.V., and Nadezhdina, E.S. (2013). Association of nucleus and centrosome: magnet or velcro? Cell biology international 37, 95-104

Cukierman, E., Pankov, R., Stevens, D.R., and Yamada, K.M. (2001). Taking cell-matrix adhesions to the third dimension. Science 294, 1708-1712

Deramaudt, T.B., Dujardin, D., Hamadi, A., Noulet, F., Kolli, K., De Mey, J., Takeda, K., and Ronde, P. (2011). FAK phosphorylation at Tyr-925 regulates cross-talk between focal adhesion turnover and cell protrusion. Mol Biol Cell 22, 964-975

Deramaudt, T.B., Dujardin, D., Noulet, F., Martin, S., Vauchelles, R., Takeda, K., and Ronde, P. (2014). Altering FAK-paxillin interactions reduces adhesion, migration and invasion processes. PloS one $\mathbf{9}$, e92059

Dohner, K., Wolfstein, A., Prank, U., Echeverri, C., Dujardin, D., Vallee, R., and Sodeik, B. (2002). Function of dynein and dynactin in herpes simplex virus capsid transport. Mol Biol Cell 13, 2795-2809

Doyle, A.D., Wang, F.W., Matsumoto, K., and Yamada, K.M. (2009). One-dimensional topography underlies three-dimensional fibrillar cell migration. J Cell Biol 184, 481-490

Dujardin, D.L., Barnhart, L.E., Stehman, S.A., Gomes, E.R., Gundersen, G.G., and Vallee, R.B. (2003). A role for cytoplasmic dynein and LIS1 in directed cell movement. J Cell Biol 163, 1205-1211

Efimov, A., Schiefermeier, N., Grigoriev, I., Ohi, R., Brown, M.C., Turner, C.E., Small, J.V., and Kaverina, I. (2008). Paxillin-dependent stimulation of microtubule catastrophes at focal adhesion sites. J Cell Sci 121, 196-204

Elric, J., and Etienne-Manneville, S. (2014). Centrosome positioning in polarized cells: common themes and variations. Experimental cell research 328, 240-248

Etienne-Manneville, S. (2013). Microtubules in cell migration. Annual review of cell and developmental biology 29, 471-499

Etienne-Manneville, S., and Hall, A. (2001). Integrin-mediated activation of Cdc42 controls cell polarity in migrating astrocytes through PKCzeta. Cell 106, 489-498

Etienne-Manneville, S., Manneville, J.B., Nicholls, S., Ferenczi, M.A., and Hall, A. (2005). Cdc42 and Par6-PKCzeta regulate the spatially localized association of DIg1 and APC to control cell polarization. J Cell Biol 170, 895-901

Ezratty, E.J., Partridge, M.A., and Gundersen, G.G. (2005). Microtubule-induced focal adhesion disassembly is mediated by dynamin and focal adhesion kinase. Nature cell biology 7, 581-590

Friedl, P., Wolf, K., and Lammerding, J. (2011). Nuclear mechanics during cell migration. Curr Opin Cell Biol 23, 55-64 
Garces, J.A., Clark, I.B., Meyer, D.I., and Vallee, R.B. (1999). Interaction of the p62 subunit of dynactin with Arp1 and the cortical actin cytoskeleton. Current biology 9, 1497-1500

Gerashchenko, M.V., Chernoivanenko, I.S., Moldaver, M.V., and Minin, A.A. (2009). Dynein is a motor for nuclear rotation while vimentin IFs is a "brake". Cell biology international 33, 1057-1064

Gomes, E.R., Jani, S., and Gundersen, G.G. (2005). Nuclear movement regulated by Cdc42, MRCK, myosin, and actin flow establishes MTOC polarization in migrating cells. Cell 121, 451-463

Houben, F., Willems, C.H.M.P., Declercq, I.L.J., Hochstenbach, K., Kamps, M.A., Snoeckx, L.H.E.H., Ramaekers, F.C.S., and Broers, J.L.V. (2009). Disturbed nuclear orientation and cellular migration in Atype lamin deficient cells. Biochimica et biophysica acta 1793, 312-324

Illc, D., Furuta, Y., Kanazawa, S., Takeda, N., Sobue, K., Nakatsuji, N., Nomura, S., Fujimoto, J., Okada, M., Yamamoto, T., et al. (1995). Reduced Sell Motility and Enhanced Focal Adhesion Contact Formation in Cells from Fak-Deficient Mice. Nature 377, 539-544

Kaverina, I., Krylyshkina, O., and Small, J.V. (1999). Microtubule targeting of substrate contacts promotes their relaxation and dissociation. J Cell Biol 146, 1033-1044

King, S.J., Brown, C.L., Maier, K.C., Quintyne, N.J., and Schroer, T.A. (2003). Analysis of the dyneindynactin interaction in vitro and in vivo. Mol Biol Cell 14, 5089-5097

Krendel, M., Zenke, F.T., and Bokoch, G.M. (2002). Nucleotide exchange factor GEF-H1 mediates cross-talk between microtubules and the actin cytoskeleton. Nature cell biology 4, 294-301

Le Boeuf, F., Houle, F., Sussman, M., and Huot, J. (2006). Phosphorylation of focal adhesion kinase (FAK) on Ser732 is induced by rho-dependent kinase and is essential for proline-rich tyrosine kinase2-mediated phosphorylation of FAK on Tyr407 in response to vascular endothelial growth factor. Mol Biol Cell 17, 3508-3520

Lee, J.S.H., Chang, M.I., Tseng, Y., Wirtz, D., Wirtz, D., and Lee, J.S.H. (2005). Cdc42 mediates nucleus movement and MTOC polarization in Swiss 3 T3 fibroblasts under mechanical shear stress. Mol Biol Cell 16, 871-880

Lee, J.S.H., Hale, C.M., Panorchan, P., Khatau, S.B., George, J.P., Tseng, Y., Stewart, C.L., Hodzic, D., and Wirtz, D. (2007). Nuclear lamin A/C deficiency induces defects in cell mechanics, polarization, and migration. Biophys J 93, 2542-2552

Levy, J.R., and Holzbaur, E.L. (2008). Dynein drives nuclear rotation during forward progression of motile fibroblasts. J Cell Sci 121, 3187-3195

Li, C.H., and Tam, P.K.S. (1998). An iterative algorithm for minimum cross entropy thresholding. Pattern Recognition Letters 19, 771-776

Luxton, G.W., Gomes, E.R., Folker, E.S., Vintinner, E., and Gundersen, G.G. (2010). Linear arrays of nuclear envelope proteins harness retrograde actin flow for nuclear movement. Science 329, 956959

Luxton, G.W., Gomes, E.R., Folker, E.S., Worman, H.J., and Gundersen, G.G. (2011). TAN lines: a novel nuclear envelope structure involved in nuclear positioning. Nucleus 2, 173-181 
Luxton, G.W., and Gundersen, G.G. (2011). Orientation and function of the nuclear-centrosomal axis during cell migration. Curr Opin Cell Biol 23, 579-588

Maninova, M., Iwanicki, M.P., and Vomastek, T. (2014). Emerging role for nuclear rotation and orientation in cell migration. Cell Adhes Migr 8, 42-48

Maninova, M., Klimova, Z., Parsons, J.T., Weber, M.J., Lwanicki, M.P., and Vomastek, T. (2013). The Reorientation of Cell Nucleus Promotes the Establishment of Front-Rear Polarity in Migrating Fibroblasts. J Mol Biol 425, 2039-2055

Manneville, J.B., Jehanno, M., and Etienne-Manneville, S. (2010). Dlg1 binds GKAP to control dynein association with microtubules, centrosome positioning, and cell polarity. J Cell Biol 191, 585-598

Melkonian, K.A., Maier, K.C., Godfrey, J.E., Rodgers, M., and Schroer, T.A. (2007). Mechanism of dynamitin-mediated disruption of dynactin. J Biol Chem 282, 19355-19364

Miller, P.M., Folkmann, A.W., Maia, A.R., Efimova, N., Efimov, A., and Kaverina, I. (2009). Golgiderived CLASP-dependent microtubules control Golgi organization and polarized trafficking in motile cells. Nature cell biology 11, 1069-1080

Mousson, A., Sick, E., Carl, P., Dujardin, D., De Mey, J., and Ronde, P. (2018). Targeting Focal Adhesion Kinase Using Inhibitors of Protein-Protein Interactions. Cancers $\mathbf{1 0}$

Osorio, D.S., and Gomes, E.R. (2014). Connecting the nucleus to the cytoskeleton for nuclear positioning and cell migration. Advances in experimental medicine and biology 773, 505-520

Otsu, N. (1979). A Threshold Selection Method from Gray-Level Histograms. IEEE Transactions on Systems, Man, and Cybernetics 9, 62-66

Palazzo, A.F., Eng, C.H., Schlaepfer, D.D., Marcantonio, E.E., and Gundersen, G.G. (2004). Localized stabilization of microtubules by integrin- and FAK-facilitated Rho signaling. Science $\mathbf{3 0 3}, \mathbf{8 3 6 - 8 3 9}$

Palazzo, A.F., Joseph, H.L., Chen, Y.J., Dujardin, D.L., Alberts, A.S., Pfister, K.K., Vallee, R.B., and Gundersen, G.G. (2001). Cdc42, dynein, and dynactin regulate MTOC reorientation independent of Rho-regulated microtubule stabilization. Curr Biol 11, 1536-1541

Park, A.Y.J., Shen, T.L., Chien, S., and Guan, J.L. (2009). Role of Focal Adhesion Kinase Ser-732 Phosphorylation in Centrosome Function during Mitosis. J Biol Chem 284, 9418-9425

Plotnikov, S.V., Pasapera, A.M., Sabass, B., and Waterman, C.M. (2012). Force fluctuations within focal adhesions mediate ECM-rigidity sensing to guide directed cell migration. Cell 151, 1513-1527

Pouthas, F., Girard, P., Lecaudey, V., Ly, T.B., Gilmour, D., Boulin, C., Pepperkok, R., and Reynaud, E.G. (2008). In migrating cells, the Golgi complex and the position of the centrosome depend on geometrical constraints of the substratum. J Cell Sci 121, 2406-2414

Prager-Khoutorsky, M., Lichtenstein, A., Krishnan, R., Rajendran, K., Mayo, A., Kam, Z., Geiger, B., and Bershadsky, A.D. (2011). Fibroblast polarization is a matrix-rigidity-dependent process controlled by focal adhesion mechanosensing. Nature cell biology 13, 1457-1465 
Robinson, J.T., Wojcik, E.J., Sanders, M.A., McGrail, M., and Hays, T.S. (1999). Cytoplasmic dynein is required for the nuclear attachment and migration of centrosomes during mitosis in Drosophila. J Cell Biol 146, 597-608

Rosse, C., Boeckeler, K., Linch, M., Radtke, S., Frith, D., Barnouin, K., Morsi, A.S., Hafezparast, M., Howell, M., and Parker, P.J. (2012). Binding of dynein intermediate chain 2 to paxillin controls focal adhesion dynamics and migration. J Cell Sci 125, 3733-3738

Schlessinger, K., McManus, E.J., and Hall, A. (2007). Cdc42 and noncanonical Wnt signal transduction pathways cooperate to promote cell polarity. J Cell Biol 178, 355-361

Schmoranzer, J., Fawcett, J.P., Segura, M., Tan, S., Vallee, R.B., Pawson, T., and Gundersen, G.G. (2009). Par3 and dynein associate to regulate local microtubule dynamics and centrosome orientation during migration. Current biology 19, 1065-1074

Schutze, K., Maniotis, A., and Schliwa, M. (1991). The position of the microtubule-organizing center in directionally migrating fibroblasts depends on the nature of the substratum. Proc Natl Acad Sci U S A 88, 8367-8371

Serrels, B., Sandilands, E., and Frame, M.C. (2011). Signaling of the direction-sensing FAK/RACK1/PDE4D5 complex to the small GTPase Rap1. Small GTPases 2, 54-61

Serrels, B., Sandilands, E., Serrels, A., Baillie, G., Houslay, M.D., Brunton, V.G., Canel, M., Machesky, L.M., Anderson, K.I., and Frame, M.C. (2010). A complex between FAK, RACK1, and PDE4D5 controls spreading initiation and cancer cell polarity. Current biology 20, 1086-1092

Shima, D.T., Haldar, K., Pepperkok, R., Watson, R., and Warren, G. (1997). Partitioning of the Golgi apparatus during mitosis in living HeLa cells. J Cell Biol 137, 1211-1228

Small, J.V., and Kaverina, I. (2003). Microtubules meet substrate adhesions to arrange cell polarity. Curr Opin Cell Biol 15, 40-47

Stehbens, S., and Wittmann, T. (2012). Targeting and transport: how microtubules control focal adhesion dynamics. J Cell Biol 198, 481-489

Tilghman, R.W., and Parsons, J.T. (2008). Focal adhesion kinase as a regulator of cell tension in the progression of cancer. Seminars in cancer biology 18, 45-52

Tomar, A., Lim, S.T., Lim, Y., and Schlaepfer, D.D. (2009). A FAK-p120RasGAP-p190RhoGAP complex regulates polarity in migrating cells. J Cell Sci 122, 1852-1862

Wang, H.B., Dembo, M., Hanks, S.K., and Wang, Y. (2001). Focal adhesion kinase is involved in mechanosensing during fibroblast migration. Proc Natl Acad Sci U S A 98, 11295-11300

Waterman-Storer, C.M., Worthylake, R.A., Liu, B.P., Burridge, K., and Salmon, E.D. (1999). Microtubule growth activates Rac1 to promote lamellipodial protrusion in fibroblasts. Nature cell biology 1, 45-50

Wen, Y., Eng, C.H., Schmoranzer, J., Cabrera-Poch, N., Morris, E.J., Chen, M., Wallar, B.J., Alberts, A.S., and Gundersen, G.G. (2004). EB1 and APC bind to mDia to stabilize microtubules downstream of Rho and promote cell migration. Nature cell biology 6, 820-830 
Wolfenson, H., Lubelski, A., Regev, T., Klafter, J., Henis, Y.I., and Geiger, B. (2009). A role for the juxtamembrane cytoplasm in the molecular dynamics of focal adhesions. PloS one 4, e4304

Xie, Z.G., and Tsai, L.H. (2004). Cdk5 phosphorylation of FAK regulates centrosome-associated microtubules and neuronal migration. Cell Cycle 3, 108-110

Yvon, A.-M.C., Walker, J.W., Danowski, B., Fagerstrom, C., Khodjakov, A., and Wadsworth, P. (2002). Centrosome reorientation in wound-edge cells is cell type specific. Mol Biol Cell 13, 1871-1880

Zhang, X., Lei, K., Yuan, X., Wu, X., Zhuang, Y., Xu, T., Xu, R., and Han, M. (2009). SUN1/2 and Syne/Nesprin-1/2 complexes connect centrosome to the nucleus during neurogenesis and neuronal migration in mice. Neuron 64, 173-187 


\section{Figure Legends}

Figure 1: FAK is required for centrosome/Golgi orientation in migrating mouse fibroblasts.

Measurement of the polarity angles $(\alpha)$ formed between cell migration (green) and polarization (red) axis in different types of fibroblasts. A) Schematic representation of the method used to calculate $\alpha$. The migration axis is defined as a line perpendicular to the wound. The polarity axis is defined by the line passing through the centroid of the nucleus and through either the centroid of the Golgi, or the centrosome closest to the wound. Measurements were made on cells subjected to wound healing assays, fixed with 3\% PFA 4h after wounding and processed for immunofluorescence. The nuclei were stained with DAPI (blue). FAK ${ }^{-/}$MEFs expressing FAK-EGFP were localized using the EGFP signal (green), centrosomes were stained using an anti $\gamma$-tubulin antibody (yellow dots), and the Golgi apparatus was stained using anti GM130 antibody (red). The white lines represent the position of the leading edge of migrating cells. Scale bar: $10 \mu \mathrm{m}$. B) Mean $\alpha$ values using the centroid of the Golgi apparatus as polarity marker. $\mathrm{FAK}^{-/-}$cells displayed significantly higher $\alpha(54.1$ $\pm 4.8^{\circ} ; 9$ experiments, 983 cells $)$ as compared to NIH $3 \mathrm{~T} 3$ cells $\left(19.2 \pm 0.7^{\circ} ; 3\right.$ experiments, 386 cells), wt MEFs (35.6 $\pm 3^{\circ} ; 5$ experiments, 372 cells), and FAK ${ }^{-/-}$MEFs expressing FAKEGFP $\left(26.8 \pm 4.9^{\circ} ; 14\right.$ experiments, 567 cells $)$. FAK ${ }^{-/}$cells expressing FAK S732A-EGFP displayed significantly higher $\alpha\left(44.3 \pm 3.9^{\circ}, 7\right.$ experiments, 312 cells) compared to FAK expressing cells. wt MEFs also polarized significantly less efficiently than NIH 3T3 cells and $\mathrm{FAK}^{-/}$cells expressing FAK-EGFP. A significant difference was also detected between $\mathrm{FAK}^{-/-}$ cells and FAK S732A-EGFP expressing cells, suggesting a partial recovery of the orientation of the Golgi with the S732A-EGFP construct. C) Mean percentage of polarized cells from the data set in (B). Cells with $\alpha>45^{\circ}$ were filed as non-polarized cells. A significantly lower proportion of $\mathrm{FAK}^{-/}$cells $(54.3 \pm 4.2 \%)$, or FAK S732A-EGFP $(64.5 \pm 6.2 \%)$ expressing cells, display polarized Golgi as compared to NIH 3T3 cells $(91.7 \pm 2.5 \%)$, wt MEFs, and FAK $^{-/}$MEFs expressing FAK-EGFP $(81.1 \pm 6.9 \%)$. wt MEFs $(74.3 \pm 5.7 \%)$ also polarized also significantly less frequently than NIH $3 \mathrm{~T} 3$ cells.

Figure 2: Dynein co-immunoprecipitates with FAK and paxillin in MEFs and the association of Dynein with FAK is required for proper polarization.

A) The upper 3 panels are representative western blots showing co-immunoprecipitation experiments using wt and $\mathrm{FAK}^{-/}$cell extracts. Antibodies used for immunoprecipitation are indicated on the left side of the figure (IP), and antibodies used to reveal the western blot are indicated on the right side (IB). The lower 3 panels are western blots probing for the different proteins studied in the cell extracts used for the immunoprecipitations. B) Representative FAK immunoprecipitation using FAK $^{-/-}$cell extracts expressing either FAK-EGFP or FAK S732A-EGFP and probed by either FAK or DIC. C) Quantification of the level of dynein coimmunoprecipitating with FAK S732A-EGFP $(0.56 \pm 0.12)$ normalized on FAK-EGFP. Values are from 3 independent experiments. 
Figure 3: FAK is required for proper enrichment of dynein, APC, and GKAP at the leading edge of migrating cells.

A) Confluent MEF wt and MEF FAK ${ }^{-/}$cells were fixed $3 \mathrm{~h}$ after wounding using methanol (dynein), or paraformaldehyde (APC and GKAP) fixation, followed by an immunostaining and imaged via either standard (EPI) or TIRF microscopy. Immunostainings using DIC, APC or GKAP directed antibodies reveal an enrichment of the proteins at the leading edge (arrows). This enrichment is reduced in $\mathrm{FAK}^{-/}$cells. Scale bar: $10 \mu \mathrm{m}$. B) Quantification of the relative enrichment of dynein, APC and GKAP at cell leading edge. The mean signal intensities, corrected from background, were measured at leading edge cell protrusions and in the whole cell to calculate a ratio. C) Quantification of the percentage of cells displaying enrichment of dynein, APC and GKAP proteins at the leading edge. D) wt MEFs were immunostained for GKAP (red) and DIC (green). Scale bar: $5 \mu \mathrm{m}$. Upper panels are magnified views of the indicated region of interest (Scale bar : $1 \mu \mathrm{m}$ ). E) Cells co-expressing low levels (10\% population displaying the lowest expression level of dynamitin-EGFP among all expressing cells) of EGFP tagged versions of DIC or dynamitin, and mCherry tagged vinculin, were imaged within $3 \mathrm{~h}$ after wounding. DIC enrichment at the leading edge (top row, arrows and boxed region of interest) was observed in $90 \%$ of NIH 3T3 $(n=15)$ or wt MEF $(n=14)$ cells, but not in FAK ${ }^{-/-}$cells $(n=20)$. Scale bars: $10 \mu \mathrm{m}$. Magnified views of the regions of interest (Scale bars: $2 \mu \mathrm{m}$ ) are also presented, along with a line intensity profile quantification (white line in magnified views). Green arrows represent dynein spots present at the level of focal adhesions. Cells of the two bottom rows were transfected only by EGFP tagged versions of DIC or dynamitin and were fixed $3 \mathrm{~h}$ after wounding using paraformaldehyde/glutaraldehyde fixation followed by tubulin immunostaining or actin (phalloidin) staining; Scale bars: $5 \mu \mathrm{m}$. 
Figure 4: FAK regulates the dynamics of dynactin colocalization with focal adhesions.

2 to 3 hours after wounding, MEFs coexpressing dynamitin-EGFP and mCherry-vinculin or FAK-mCherry were monitored using time-lapse video TIRF microscopy every 6 seconds during 10 minutes (100 frames). For quantifications, the mean values from the 100 frames were calculated for 5 to 7 representative cells. A) Representative images showing the distribution of dynamitin (green) and vinculin (red) in living wt (upper panels) , FAK ${ }^{-/}$ (middle panels), or FAK-mCherry expressing FAK ${ }^{-/-}$MEFs are represented. The right panels show colocalization (white areas) between dynamitin and vinculin or FAK-mCherry. The white line represents the separation between the front $\left(\mathrm{F}\right.$, front $1 / 3^{\text {rd }}$ of total cell length) and the rear $(\mathrm{R})$ of cells used for sub-region analysis, and the thin white curve represents the edge of the wound. Dashed yellow lines represent the zones selected for the kymographs. Scale bar: $5 \mu \mathrm{m}$. B) Quantification of the mean percentage of sub-region surface (front or rear) displaying dynamitin positive signal per frame on wt ( F : $1.31 \pm 0.77 \%$; $\mathrm{R}: 0.005 \pm 0.006$ $\%)$, FAK $^{-/-}(\mathrm{F}: 1.27 \pm 0.93 \%$; R : $0.82 \pm 0.44 \%)$ or FAK-mCherry expressing FAK $^{-/-}$MEFs ( $F: 0.71 \pm 0.31 \% ; R: 0.11 \pm 0.09 \%$ ). The enrichment of dynamitin is statistically significant between the front and the rear of only wt MEFs and $\mathrm{FAK}^{-/}$cells expressing FAK-mCherry. C) Quantification of the mean percentage of sub-region surface (front or rear) having both dynamitin and vinculin positive signal per frame on wt MEFs (F : $0.77 \pm 0.48 \%$; $\mathrm{R}: 0.004 \pm$ $0.003 \%)$, FAK $^{-/-}(\mathrm{F}: 0.12 \pm 0.07 \%$; R : $0.07 \pm 0.05 \%)$ or FAK-mCherry expressing FAK $^{-/-}$ MEFs ( $F: 0.11 \pm 0.13 \%$; $\mathrm{R}: 0.007 \pm 0.007 \%$ ). The enrichment of colocalization is statistically significant between the front and the rear of only wt MEFs and $\mathrm{FAK}^{-/}$cells expressing FAK-mCherry. D) Kymographs displaying the evolution of segmented signal (green : dynamitin; red : vinculin or FAK-mCherry, white: colocalization) along the yellow dashed lines ( $a, b, c, d$, e, and f) drawn in panel (A) over time. Spots are indicative of the dynamic nature of dynamitin signal, and white lines are indicative of longer dwell times. These lines are clearly visible at the front of wt and FAK-mCherry expressing FAK ${ }^{-/}$MEFs (arrows). Scale bar x axis (time) : 2 min ; Scale bar y axis: $1 \mu \mathrm{m}$. E) Quantification of the mean percentage of dwell times superior to 3 frames (12 seconds) of colocalizing signals at the front or rear of wt $(\mathrm{F}: 15.64 \pm 10 \%$; $\mathrm{R}: 1.63 \pm 3.24 \%), \mathrm{FAK}^{-/}(\mathrm{F}: 6.37 \pm 2.46 \%$; $\mathrm{R}$ : $6.73 \pm 3.52 \%$ ) or FAK-mCherry expressing FAK $^{-/}$MEFs (F : $8.3 \pm 2.08 \%$; R : $2.2 \pm 4.42$ $\%)$. Longer dwell times enrichment is statistically significant between the front and the rear of only wt MEFs and $\mathrm{FAK}^{-/}$cells expressing FAK-mCherry. 
Figure 5: Contribution of nucleus rearward movement to Golgi polarization in living mouse fibroblasts.

Confluent monolayers of cells expressing either NAGFP or mCherry-GT and FAK-EGFP or FAK S732A-EGFP were wounded, and non-already polarized cells at the front were monitored to visualize phase contrast and fluorescent Golgi signal. The time lapse microcopy sequence started within 30 minutes after wounding, and was performed during 5 hours with one picture every 5 minutes (MEFs) or 10 minutes (NIH3T3). Shown results for the following figures were obtained from 3 to 4 independent experiments analyzing 24 (NIH3T3), 17 (wt MEFs), 32 (FAK ${ }^{--}$), 33 (FAK S732A-EGFP), and 47 (FAK-EGFP) cells. A) Measurement of the positions of the cell front $(\mathrm{F})$, the cell rear $(\mathrm{R})$, as well as the centroid of the nucleus $(\mathrm{N})$ and the Golgi $(\mathrm{G})$ before and after polarization. B) Quantification of nucleus displacement during polarization as a percentage of total cell length for NIH $3 \mathrm{~T} 3(-8.3 \pm 7.4 \%)$, wt MEFs $(-10.5 \pm 15.7 \%)$, MEFs expressing FAK-EGFP $(-13.0 \pm 11.6 \%)$ or FAK S732A-EGFP $(-7.9 \pm$ $10.9 \%)$, or $\mathrm{FAK}^{-/}$cells $(2.17 \pm 13.4 \%)$. C) Quantification of Golgi displacement during polarization as a percentage of total cell length for NIH $3 \mathrm{~T} 3(11.6 \pm 10.8 \%)$, wt MEFs $(10.6$ $\pm 30.7 \%)$, MEFs expressing FAK-EGFP $(14.1 \pm 12.8 \%)$ or FAK S732A-EGFP $(6.7 \pm 19.1 \%)$, or FAK ${ }^{-/-}$cells $(-8.1 \pm 17.4 \%)$. D) Percentage of cells that succeeded in orienting their Golgi toward the migratory front within the time frame of the video. A significant decrease in the frequency of orientation is visible for $\mathrm{FAK}^{-/-}$and cells expressing FAK S732A-EGFP (respectively $33.4 \pm 12.1 \%$, and $42.2 \pm 9.7 \%$ ) compared to NIH $3 \mathrm{~T} 3(100 \pm 0 \%$ ), wt MEF $(77.8 \pm 19.2)$, and FAK $^{-/-}$expressing FAK-EGFP $(90.8 \pm 8.3 \%)$. All combinations of comparison were tested via ANOVA, and only statistically significant differences are depicted. Scale bars: $5 \mu \mathrm{m}$.

\section{Figure 6: Measurement of nucleus rotation events.}

A) NRs were monitored using nucleoli as nucleus orientation markers (yellow numbers) allowing to draw an initial axis (yellow dotted arrows) and their subsequent evolution (yellow plain arrow). NR events were defined as persistent changes in the orientation of the nucleolus during at least 3 consecutive frames with a final amplitude superior to $10^{\circ}$. Scale bars: $5 \mu \mathrm{m}$. B) Percentage of cells that displayed NR during the time frame of the video. $\mathrm{FAK}^{-/-}$MEFs have significantly reduced frequencies of NR $(26.2 \pm 5.7 \%)$ compared to NIH 3T3 $(62.1 \pm$ $4.8 \%$ ), wt MEFs (44.4 $\pm 9.6 \%$ ), and MEFs expressing FAK-EGFP $(52.8 \pm 3.4 \%)$ or FAK S732A-EGFP $(46.4 \pm 3.2 \%)$. The same times laps recordings and cell numbers as Figure 5 were used for this analysis. All combinations of comparison were tested via ANOVA, and only statistically significant differences are depicted. 
Figure 7: FAK but not its interaction with dynein, is required for NR associated Golgi orientation.

A) Representative behavior of FAK-EGFP or FAK S732A-EGFP expressing $\mathrm{FAK}^{-/}$cells displaying nuclear rotation associated Golgi orientation. Golgi orientation relative to the nucleus was monitored by following the evolution of the axis between the nucleus and the Golgi before (red doted axis) and after (red plain arrow) polarization. Green inserts show the expression of the FAK construct in the monitored cells. Scale bars: $5 \mu \mathrm{m}$. A graphical representation displaying the time course measurements of the polarization angles of the nucleus (black curve) and the Golgi (red curve), as well as the positioning of the nucleus (blue curve) and the Golgi (yellow curve) as a percentages of total cell length, is shown for each illustrated cell. B) Percentage of cells that displayed Golgi orientation associated to NR during the time frame of the video. $\mathrm{FAK}^{-/-} \mathrm{MEFs}$ have significantly reduced frequencies of these events $(9.3 \pm 9.3 \%)$ compared to NIH $3 \mathrm{~T} 3(49.5 \pm 6.3 \%)$, wt MEFs $(38.9 \pm 9.6 \%)$, and MEFs expressing FAK-EGFP $(32.7 \pm 12.5 \%)$ or FAK S732A-EGFP $(36.7 \pm 7.6 \%)$. The same times laps recordings and cell numbers as Figure 5 were used for this analysis. All combinations of comparison were tested via ANOVA, and only statistically significant differences are depicted.

Figure 8: FAK interaction with dynein is required for NR independent orientation of the Golgi.

A) Representative behavior of cells displaying Golgi orientation that are independent of NR events (NIH 3T3, FAK-EGFP). Scale bars: $5 \mu \mathrm{m}$. A graphical representation displaying the time course measurements of the polarization angles of the nucleus (black curve) and the Golgi (red curve), as well as the positioning of the nucleus (blue curve) and the Golgi (yellow curve) as a percentages of total cell length, is shown for each illustrated cell. B) Percentage of cells that displayed Golgi orientation independently of NR during the time frame of the video. $\mathrm{FAK}^{-/}$and FAK S732A-EGFP expressing MEFs have significantly reduced frequencies of orientation (respectively $9.3 \pm 9.3 \%$ and $8.6 \pm 8.3 \%$ ) compared to NIH 3 T3 $(71 \pm 4.2 \%$ ), wt MEFs (55.4 $\pm 25.3 \%)$, and MEFs expressing FAK-EGFP (57.3 $\pm 9.1 \%)$. The same times laps recordings and cell numbers as Figure 5 were used for this analysis. All combinations of comparison were tested via ANOVA, and only statistically significant differences are depicted. 


\section{Supplemental Material}

Figure S1: Non phosphorylatable (S732A) FAK locates at focal adhesion and is properly phosphorylated on Y397, Y861 and Y925.

Non phosphorylatable (S732A) FAK sequence was generated via PCR and its functionality was analyzed using: A) Western blot analysis of cell extracts after expression of the wt and S732A version of FAK. For each experiment, the same blot was revealed using either an antibody recognizing FAK phosphorylated on the residues Y397 (upper panels), Y861 (middle panels), or Y925 (lower panels), or total FAK. $\alpha$-tubulin loading controls are provided for each blot. FAK in MEF extracts and EGFP tagged constructs are detected at the expected molecular weights. . B) Ratiometric quantifications of band intensities from the previous experiments. The mean intensity ratios (phosphorylated/total FAK normalized with FAK-EGFP as reference) from 3 independent experiments are displayed and no significant effect was observed on the phosphorylation level of Y397 $(1.1 \pm 0.09)$, Y861 $(0.98 \pm 0.13)$, and Y925 (0.89 \pm 0.06$)$ residues.). C) Immunofluorescence experiment displaying EGFP signal (left panels, green in overlays), FAK Y397 phosphorylation (middle panel, red in overlays), and colored overlays of the signal showing also the nucleus (DAPI, blue). FAKEGFP and FAK S732A-EGFP display detectable Y397 phosphorylation at focal adhesions. Scale bar, $10 \mu \mathrm{m}$. D) Quantification of the mean grey value of individual focal adhesions for total FAK (EGFP) and phosphorylated Y397 FAK (8 to 10 cells for each construct). E) Statistical analysis of the mean slope values obtained from the different cells of preceding quantification showing that FAK-EGFP and FAK S732A-EGFP cells have no significant difference in Y397 phosphorylation at focal adhesions $(2.9 \pm 0.44$ and $2.7 \pm 1.1$ for FAKEGFP and FAK S732A-EGFP respectively). F) Representative western blots showing DIC, $\mathrm{APC}$, or GKAP (upper panels) in $\mathrm{FAK}^{-/}$and wt MEFs, as well as tubulin loading controls (lower panels).

\section{Movie S2:}

Live-cell imaging of a wt MEF co-transfected with plasmids encoding for DIC-EGFP and mCherry-vinculin. Time-lapse TIRF images were collected every 6 seconds and are displayed at $10 \mathrm{fps}$.

\section{Movie S3:}

Live-cell imaging of a $\mathrm{FAK}^{-/-} \mathrm{MEF}$ co-transfected with plasmids encoding for DIC-EGFP and mCherry-vinculin. Time-lapse TIRF images were collected every 6 seconds and are displayed at $10 \mathrm{fps}$.

\section{Movie S4:}

Live-cell imaging of a wt MEF co-transfected with plasmids encoding for dynamitin-EGFP and mCherry-vinculin. Time-lapse TIRF images were collected every 6 seconds and are displayed at $10 \mathrm{fps}$.

\section{Movie S5:}

Live-cell imaging of a $\mathrm{FAK}^{-/-} \mathrm{MEF}$ co-transfected with plasmids encoding for dynamitinEGFP and mCherry-vinculin. Time-lapse TIRF images were collected every 6 seconds and are displayed at $10 \mathrm{fps}$. 


\section{Movie S6:}

Live-cell imaging of a $\mathrm{FAK}^{-/-} \mathrm{MEF}$ co-transfected with plasmids encoding for dynamitinEGFP and FAK-mCherry. Time-lapse TIRF images were collected every 6 seconds and are displayed at $10 \mathrm{fps}$.

\section{Movie S7:}

Representative NIH3T3 cell displaying Golgi orientation occurring independently of NR. Cells were transfected with plasmids encoding for the NAGFP Golgi marker and recorded by time-lapse wide field fluorescence and phase contrast microscopy ( 1 image every $10 \mathrm{~min}$ displayed at $7 \mathrm{fps})$.

\section{Movie S8:}

Representative $\mathrm{FAK}^{-/-} \mathrm{MEF}$ cell displaying no Golgi orientation. Cells were transfected with plasmids encoding for the mCherry-GT Golgi marker and recorded by time-lapse wide field fluorescence and phase contrast microscopy ( 1 image every 5 min displayed at $14 \mathrm{fps}$ ).

\section{Movie S9:}

Representative $\mathrm{FAK}^{-/-}$MEF cell expressing FAK S732A-EGFP and displaying no final Golgi orientation. Cells were co-transfected with plasmids encoding for the mCherry-GT Golgi marker and FAK S732A-eEGFP (not shown), and recorded by time-lapse wide field fluorescence and phase contrast microscopy (1 image every 5 min displayed at $14 \mathrm{fps}$ ).

\section{Movie S10:}

Representative NIH3T3 cell displaying Golgi orientation associated with NR. Cells were transfected with plasmids encoding for the NAGFP Golgi marker and recorded by time-lapse wide field fluorescence and phase contrast microscopy (1 image every $10 \mathrm{~min}$ displayed at 7 fps).

\section{Movie S11:}

Representative $\mathrm{FAK}^{-/-}$MEF cell expressing FAK-EGFP and displaying Golgi orientation associated with NR. Cells were co-transfected with plasmids encoding for the mCherry-GT Golgi marker and FAK-eEGFP (not shown) and recorded by time-lapse wide field fluorescence and phase contrast microscopy (1 image every 5 min displayed at $14 \mathrm{fps}$ ).

\section{Movie S12:}

Representative $\mathrm{FAK}^{-/-}$MEF cell expressing FAK S732A-EGFP and displaying Golgi orientation associated with NR. Cells were co-transfected with plasmids encoding for the mCherry-GT Golgi marker and FAK S732A-EGFP (not shown), and recorded by time-lapse wide field fluorescence and phase contrast microscopy (1 image every 5 min displayed at 14 fps).

\section{Movie S13:}

Representative $\mathrm{FAK}^{-/-}$MEF cell expressing FAK-EGFP and displaying Golgi orientation occurring independently of NR. Cells were co-transfected with plasmids encoding for the mCherry-GT Golgi marker and FAK-EGFP (not shown) and recorded by time-lapse wide field fluorescence and phase contrast microscopy ( 1 image every 5 min displayed at $14 \mathrm{fps}$ ). 